\title{
Generalizability of the Results of Cardiovascular Outcome Trials of Glucagon-Like Peptide 1 Receptor Agonists in Chinese Patients with Type 2 Diabetes Mellitus
}

Xiaoling Cai $\cdot$ Linong Ji

Received: March 16, 2021 / Accepted: May 11, 2021 / Published online: May 28, 2021

(c) The Author(s) 2021

\section{ABSTRACT}

Introduction: This study aimed to investigate the generalizability of the results of cardiovascular outcome trials (CVOTs) of glucagon-like peptide 1 receptor agonists (GLP-1 RAs) to Chinese patients with type 2 diabetes mellitus (T2DM).

Methods: The 3B (Blood Glucose, Blood Pressure, and Blood Lipid) population, a nationally representative population of patients with T2DM in China $(n=25,411)$, was examined for eligibility of enrollment in four GLP-1 RAs CVOTs (Dulaglutide-REWIND, ExenatideEXSCEL, Liraglutide-LEADER, and SemaglutideSUSTAIN-6). We first estimated the proportion of $3 \mathrm{~B}$ population who would meet the six inclusion and exclusion (I/E) criteria, namely age, hemoglobin A1c (HbA1c), body mass index (BMI), estimated glomerular filtration rate (eGFR), history of cardiovascular disease (CVD), and antidiabetic medication, in each CVOT. Then we compared 11 baseline characteristics, namely age, gender, duration of diabetes,

Supplementary Information The online version contains supplementary material available at https:// doi.org/10.1007/s13300-021-01079-x.

X. Cai · L. Ji $(\bowtie)$

Department of Endocrinology and Metabolism,

Peking University People's Hospital, Beijing 100044,

China

e-mail: jiln@bjmu.edu.cn
HbA1c, BMI, eGFR, history of CVD, prior myocardial infarction (MI), low-density lipoprotein cholesterol (LDL-c), diastolic blood pressure (DBP), and systolic blood pressure, between the population in each CVOT and the 3B population. Lastly, we estimated the proportion of $3 \mathrm{~B}$ population that matched the characteristics in each CVOT population.

Results: On the basis of the I/E criteria, 31.1\% of the 3B population would have been eligible for enrollment in REWIND, $15.0 \%$ for SUSTAIN$6,12.9 \%$ for LEADER, and $11.3 \%$ for EXSCEL. On the basis of the baseline characteristics, REWIND most closely matched the 3B population on gender, duration of diabetes, HbA1c, DBP, LDL-c, history of CVD, and prior MI. The proportion of 3B population matching on at least eight or at least ten baseline characteristics with CVOT populations was highest for REWIND compared to other CVOTs.

Conclusion: Among the four GLP-1 RA CVOTs, the REWIND trial using once-weekly dulaglutide is most generalizable to Chinese patients with T2DM.

Trial Registration: Trial registration: NCT01128205 (www.clinicaltrials.gov).

Keywords: Cardiovascular outcome trials; Chinese; GLP-1 receptor agonist; Type 2 diabetes mellitus 


\section{Key Summary Points}

Why carry out this study?

The proportions of Chinese participants were very limited in the cardiovascular outcome trials (CVOTs) of GLP-1 RAs. It remains unknown whether results of the CVOTs, mainly from Caucasians, are applicable to the Chinese population because of ethnic differences in response to GLP-1 RAs between Asians and Caucasians.

This study evaluated the generalizability of GLP-1 RA CVOTs results to the Chinese type 2 diabetes mellitus (T2DM)

population using nationally

representative data from the 3B (Blood Glucose, Blood Pressure, and Blood Lipid) study.

\section{What was learned from the study?}

Among four GLP-1 RA CVOTs, namely REWIND, SUSTAIN-6, LEADER, and EXSCEL, REWIND for once-weekly dulaglutide is most generalizable to Chinese patients with T2DM.

Our findings are consistent with prior studies assessing the generalizability of the GLP-1 RA CVOTs to the general T2DM population in the USA and EU.

\section{DIGITAL FEATURES}

This article is published with digital features, including a summary slide, to facilitate understanding of the article. To view digital features for this article go to https://doi.org/10.6084/ m9.figshare.14566482.

\section{INTRODUCTION}

Cardiovascular disease (CVD) is the leading cause of death in patients with type 2 diabetes mellitus (T2DM) worldwide [1]. CVD risks are often considered the main indicator of safety issues in the evaluation of glucose-lowering therapies [2, 3]. For example, medications such as thiazolidinediones were found to be associated with an increased risk of serious cardiovascular events including congestive heart failure [4-6]. As a novel antidiabetic treatment, glucagon-like peptide 1 receptor agonists (GLP1 RAs) not only demonstrated an antidiabetic effect and cardiovascular safety [7-13] but also potential cardiovascular protective effects [14]. GLP-1 RAs were recommended by the American Diabetes Association (ADA) in 2020 as a preferable combination therapy after metformin for patients with diabetes and established atherosclerotic cardiovascular disease (ASCVD) or at high risk of ASCVD $[15,16]$.

Cardiovascular outcome trials (CVOTs) of GLP-1 RAs were conducted worldwide $[7,9,11,13,17-19]$; however, the proportions of Chinese participants or even Asian participants were very limited in each CVOT. The proportion of Asian population in four CVOTs, namely REWIND (Researching Cardiovascular Events with a Weekly INcretin in Diabetes) (dulaglutide; NCT01394952) [7, 8], LEADER (Liraglutide Effect and Action in Diabetes: Evaluation of cardiovascular outcome Results) (liraglutide; NCT01179048) [11, 12], SUSTAIN-6 (semaglutide; NCT01720446 [13], and EXSCEL (Exenatide Study of Cardiovascular Event Lowering) (exenatide once weekly; NCT01144338) $[9,10]$, was $1.5 \%, 9.9 \%, 8.3 \%$, and $9.8 \%$, respectively.

Although eligibility criteria for CVOTs can improve internal validity of clinical trials, they may limit the generalizability of findings to different populations recruited from heterogeneous practice settings [20]. Several studies have been conducted to examine the generalizability of results from GLP-1 RAs CVOTs to T2DM populations in the USA [21] and Europe [22], but none has been conducted in Asian patients with T2DM to our best knowledge. Ethnicity is considered one of the risk factors for diabetes and its complications including CVD [23]. It remains unknown whether results of the CVOTs, mainly from Caucasians, are applicable to Asian ethnic groups such as the Chinese 
population. Therefore, our study was the first that aimed to evaluate the generalizability of the results of GLP-1 RA CVOTs to the Chinese T2DM population.

\section{METHODS}

\section{Data Source}

We utilized the 3B study, i.e., Nationwide Assessment of Cardiovascular Risk Factors: Blood Glucose, Blood Pressure, and Blood Lipid (3B), which is representative of Chinese patients with T2DM. The 3B study was a cross-sectional, multicenter, and observational study conducted to describe CVD risk factors in Chinese patients with T2DM (the study was registered as NCT01128205 on www.clinicaltrials.gov) [24]. The 3B population was nationally representative of outpatients with T2DM enrolled at 104 hospitals in six geographic regions, namely Northeast, North, East, Northwest, Southwest, and South-Central China [25]. Patients were included in the $3 \mathrm{~B}$ population if they were 18 years or older and diagnosed with T2DM at least 6 months before screening according to the diagnostic criteria of the World Health Organization as recommended by the Chinese Diabetes Guidelines [26, 27].

A total of 25,817 patients were enrolled from August 18, 2010 to March 30, 2011 [24]. The 3B study design has been described elsewhere [24, 28-30]. Institutional review board (IRB) approval was not required for this particular study as the analysis is based on data from previously conducted studies. The study was conducted in compliance with the principles in the Declaration of Helsinki [24].

\section{Study Population}

The study population was derived from the 3B study. Patients aged 18 years or older were identified as having T2DM if they had a diagnosis of T2DM. The study population was also required to have at least one record of hemoglobin A1c (HbA1c) and estimated glomerular filtration rate (eGFR) laboratory results. Patients were excluded from the analysis if they had type 1 diabetes, were pregnant, participating in any other clinical studies, or unable to report their medical history.

\section{Cardiovascular Outcome Trials}

From the US Clinical Trials Registry [31] and previous publications, four CVOTs for GLP-1 RAs, namely REWIND (dulaglutide) [7], EXSCEL (exenatide) [9], LEADER (liraglutide) [11], and SUSTAIN-6 (semaglutide) [13], were identified and selected for this study. Three other CVOTs, namely HARMONY (albiglutide) [17], PIONEER6 (orally administered semaglutide) [18], and ELIXA (lixisenatide) [19], were excluded since albiglutide and orally administered semaglutide have not been launched in China, and ELIXA exclusively enrolled patients with T2DM hospitalized with an acute coronary syndrome event, which is very different from other CVOTs.

\section{Statistical Analysis}

The generalizability of findings from the CVOTs to the $3 \mathrm{~B}$ population was examined using three approaches. First, on the basis of the main inclusion and exclusion (I/E) criteria in each CVOT, the proportions of the $3 \mathrm{~B}$ population who would have met key I/E criteria and been enrolled in the CVOT were examined. Table 1 lists the key I/E criteria, and Table S1 in the supplementary material provides a detailed summary of how the cardiovascular criteria for each CVOT were mapped to the 3B population.

Second, the CVOT populations were compared with the $3 \mathrm{~B}$ population with respect to 11 key baseline characteristics, namely age, gender, duration of diabetes, HbA1c, body mass index (BMI), eGFR, CVD history, prior myocardial infarction (MI), low-density lipoprotein cholesterol (LDL-c), diastolic blood pressure (DBP), and systolic blood pressure (SBP). These characteristics were selected since they were reported in at least three of the selected CVOTs as well as in the 3B population. Continuous variables were reported using mean and standard 
Table 1 Patient enrollment criteria of CVOTs

\begin{tabular}{|c|c|c|c|c|}
\hline $\begin{array}{l}\text { Baseline } \\
\text { characteristics }\end{array}$ & REWIND [7] & EXSCEL [9] & LEADER [11] & SUSTAIN-6 [13] \\
\hline Cardiovascular & $\begin{array}{l}\text { ECVD, SCVD, or } \\
\text { CV risk }\end{array}$ & $70 \%$ with ECVD & ECVD or CV risk & ECVD or CV risk \\
\hline Age (years) & $\begin{array}{l}\geq 50 \text { if ECVD, } \\
\geq 55 \text { if SCVD, } \\
\text { or } \geq 60 \text { if CV risk }\end{array}$ & $\geq 18$ & $\begin{aligned} \geq & 50 \text { if ECVD or } \geq 60 \text { if } \\
& \mathrm{CV} \text { risk }\end{aligned}$ & $\begin{aligned} \geq & 50 \text { if } E C V D \text { or } \geq 60 \text { if } \\
& C V \text { risk }\end{aligned}$ \\
\hline BMI $\left(\mathrm{kg} / \mathrm{m}^{2}\right)$ & $\geq 23$ & None & None & None \\
\hline HbAlc (\%) & $\leq 9.5$ & 6.5 to 10 & $\geq 7$ & $\geq 7$ \\
\hline $\begin{array}{l}\mathrm{eGFR}(\mathrm{mL} / \\
\mathrm{min} / \\
\left.1.73 \mathrm{~m}^{2}\right)\end{array}$ & $\begin{array}{l}\geq 15 \text { and not on } \\
\text { dialysis }\end{array}$ & $\geq 30$ & $\begin{array}{c}\text { Number of patients with } \\
\text { eGFR }<30 \text { restricted } \\
\text { to } 2.5 \% \text { of population }\end{array}$ & None \\
\hline Medication & $\begin{array}{l}\text { Excluded patients } \\
\text { who use premix or } \\
\text { bolus insulin } \\
\text { or }>2 \text { oral classes } \\
\text { in } 3 \text { months prior } \\
\text { to index }\end{array}$ & $\begin{array}{l}\text { Excluded patients who } \\
\text { use insulin within } \\
2 \text { weeks of index } \\
\text { or }>3 \text { oral classes in } \\
3 \text { months prior to } \\
\text { index, or previous } \\
\text { treatment with a GLP- } \\
1 \text { receptor agonist }\end{array}$ & $\begin{array}{l}\text { Excluded patients who use } \\
\text { a GLP-1 RA, DPP4 } \\
\text { inhibitor, or } \\
\text { pramlintide within } \\
3 \text { months of index; use } \\
\text { of insulin other than } \\
\text { human NPH insulin or } \\
\text { long-acting insulin } \\
\text { analogue or premixed } \\
\text { insulin within } \\
3 \text { months prior to } \\
\text { screening }\end{array}$ & $\begin{array}{l}\text { Excluded patients who use } \\
\text { a GLP-1 RA or } \\
\text { pramlintide within } \\
3 \text { months of index; if } \\
\text { use a DPP4 inhibitor } \\
\text { within } 1 \text { month of } \\
\text { index; if being treated } \\
\text { with }>2 \text { classes of orals } \\
\text { at index }\end{array}$ \\
\hline
\end{tabular}

$B M I$ body mass index, $C V$ cardiovascular, DPP4 dipeptidyl peptidase 4, ECVD established cardiovascular disease, $e G F R$ estimated glomerular filtration rate, GLP-1 RA glucagon-like peptide 1 receptor agonist, $S C V D$ subclinical cardiovascular disease

deviation, and categorical variables were reported using frequency and percentages.

Third, we also estimated the number and proportion of patients in the $3 \mathrm{~B}$ population that met the baseline characteristics of each CVOT population, which were defined as the number of patients whose results were within the 95\% confidence interval of results in CVOTs for continuous variables (age, duration of diabetes, BMI, SBP, DBP, HbA1c, and LDL-c), and was estimated by applying the percentage of people with that characteristic in each CVOT $( \pm 5 \%)$ to the $3 \mathrm{~B}$ population for categorical variables (gender, eGFR, CVD history, and prior MI).
Furthermore, we examined the proportion of 3B population who would have met several characteristics in each CVOT population. The number and proportion of patients in the $3 \mathrm{~B}$ population who met more than 4,8 , or 10 characteristics of CVOTs were presented.

All statistical analyses were conducted using the R platform.

\section{Sensitivity Analysis}

We conducted sensitivity analysis to assess the robustness of results using five of the six I/E criteria (excluding antidiabetic medication), 
and the results are shown in Table $\mathrm{S} 2$ in the supplementary material. In addition, we examined the number and proportion of patients in the $3 \mathrm{~B}$ population matching on seven baseline characteristics (age, gender, BMI, HbA1c, eGFR, history of CVD, prior MI) of each CVOT and those who met at least two, at least four, or at least six characteristics. The results are shown in Table S3 in the supplementary material.

\section{RESULTS}

\section{Analysis Based on I/E Criteria}

A total of 25,411 patients in the $3 \mathrm{~B}$ study were included in our analyses. The proportions of the $3 \mathrm{~B}$ population that would have been enrolled into REWIND, EXSCEL, SUSTAIN-6, and LEADER if all six I/E criteria were met are shown in Fig. 1 . We found that $31.1 \%(n=7903)$ of the 3B population would have been eligible for enrollment into REWIND, $11.3 \%(n=2872)$ for EXSCEL, $12.9 \%(n=3295)$ for LEADER, and $15.0 \%(n=3822)$ for SUSTAIN-6 on the basis of all six I/E criteria. Among the four CVOTs, REWIND would have enrolled more than twice as many Chinese patients with T2DM as EXSCEL, LEADER, and SUSTAIN-6.

\section{Analysis Based on Baseline Characteristics}

Baseline characteristics of the $3 \mathrm{~B}$ population and each CVOT population are summarized in

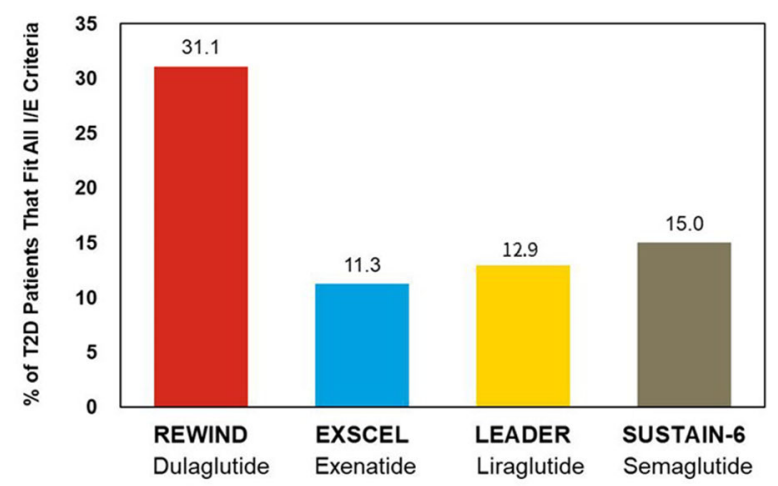

Fig. 1 Generalizability of inclusion-exclusion criteria in four GLP-1 RA CVOTs
Table 2 . The REWIND population most closely matched the 3B population on gender, duration of diabetes, HbA1c, DBP, LDL-c, CVD history, and prior MI. The EXSCEL population most closely matched 3B population on age, BMI, and SBP. The SUSTAIN-6 population most closely matched 3B population on eGFR. The LEADER population did not outperform other CVOTs when matching with $3 \mathrm{~B}$ population on any characteristics.

\section{Analysis Based on Individual Characteristics in 3B Population}

The number and percentage of individuals in the $3 \mathrm{~B}$ population who matched the 11 key baseline characteristics in each CVOT are shown in Table 3 . The numbers and percentages of $3 \mathrm{~B}$ patients matching on characteristics such as duration of diabetes, HbA1c, eGFR, LDL, SBP, and DBP were comparable across all four CVOTs. For individual characteristics, REWIND matched with $3 \mathrm{~B}$ on the highest number of characteristics across the four CVOTs. REWIND most closely matched 3B on gender, LDL-c, CVD history, and prior MI; EXSCEL most closely matched $3 \mathrm{~B}$ on age and DBP; LEADER most closely matched 3B on BMI, HbA1c, and SBP; SUSTAIN-6 most closely matched 3B on duration of diabetes, HbA1c, and eGFR. When any eight or more $(58.4 \%)$ and any 10 or more (36.4\%) of the 11 key characteristics had to be met, REWIND exceeded the other CVOTs in matching with the $3 \mathrm{~B}$ population.

\section{Sensitivity Analysis}

Consistent results were obtained for the analysis of I/E criteria including and excluding antidiabetic medication (Table $\mathrm{S} 2$ in the supplementary material). The number and proportion of individuals in the $3 \mathrm{~B}$ population matching on seven key baseline characteristics (age, gender, BMI, HbA1c, eGFR, CVD history, and prior MI) of each CVOT population are shown in Table S3 in the supplementary material. In these sensitivity analyses, REWIND performed better than other CVOTs in matching with the 3B population. 
Table 2 Baseline characteristics of 3B population and each CVOT population

\begin{tabular}{llllll}
\hline Characteristics & 3B population [24] & REWIND [7] & EXSCEL [9] & LEADER [11] & SUSTAIN-6 [13] \\
\hline Age (years) & $62.6 \pm 11.8$ & $66.2 \pm 6.5$ & $62.7 \pm 9.2^{*}$ & $64.3 \pm 7.2$ & $64.6 \pm 7.4$ \\
Gender, $n(\%)$ & & & & & \\
$\quad$ Male & $11,933(47.0 \%)$ & $5321(53.7 \%)^{*}$ & $9148(62.0 \%)$ & $6003(64.3 \%)$ & $2002(60.7 \%)$ \\
Female & $13,478(53.0 \%)$ & $4589(46.3 \%)^{*}$ & $5604(38.0 \%)$ & $3337(35.7 \%)$ & $1295(39.3 \%)$ \\
BMI $\left(\mathrm{kg} / \mathrm{m}^{2}\right)$ & $24.8 \pm 3.6$ & $32.3 \pm 5.7$ & $31.8 \pm 5.9^{*}$ & $32.5 \pm 6.3$ & $32.8 \pm 6.2$ \\
Diabetes duration (years) & $8.1 \pm 6.8$ & $10.0 \pm 7.2^{*}$ & $12 \pm 8.1$ & $12.7 \pm 8.0$ & $13.9 \pm 8.1$ \\
HbAlc $(\%)$ & $7.6 \pm 2.0$ & $7.3 \pm 1.1^{*}$ & $8.0 \pm 1.2$ & $8.7 \pm 1.5$ & $8.7 \pm 1.5$ \\
eGFR $\left(\mathrm{mL} / \mathrm{min} / 1.73 \mathrm{~m}^{2}\right)$ & & & & \\
$<60$ & $28.9 \%$ & $22.2 \%$ & $18.6 \%$ & $21.8 \%$ & $28.5 \%^{*}$ \\
$\geq 60$ & $71.1 \%$ & $77.8 \%$ & $81.4 \%$ & $78.2 \%$ & $71.5 \% *$ \\
SBP $(\mathrm{mmHg})$ & $133.0 \pm 15.7$ & $137.2 \pm 16.8$ & $135.0 \pm 15.6^{*}$ & $135.9 \pm 17.8$ & $135.6 \pm 17.2$ \\
DBP $(\mathrm{mmHg})$ & $78.8 \pm 9.0$ & $78.5 \pm 9.8^{*}$ & $80.0 \pm 11.1$ & $77.2 \pm 10.3$ & $77.0 \pm 10.0$ \\
LDL-c $(\mathrm{mg} / \mathrm{dL})$ & $109.4 \pm 35.2$ & $99.0 \pm 37.9^{*}$ & $88.0 \pm 37.0$ & $89.5 \pm 35.5$ & $82.3 \pm 45.6$ \\
CVD history, $n(\%)$ & $5751(22.6 \%)$ & $3111(31.4 \%)^{*}$ & $10,769(73.0 \%)$ & $7598(81.4 \%)$ & $1994(60.5 \%)$ \\
Prior MI, $n(\%)$ & $711(2.8 \%)$ & $1600(16.2 \%)^{*}$ & $\mathrm{NA}$ & $2864(30.7 \%)$ & $1072(32.5 \%)$ \\
\hline
\end{tabular}

This table compares the characteristics of the adult T2D population with reported characteristics from patients enrolled in each of the CVOTs. Asterisks indicate the minimal differences between adult T2D population and the CVOTs $B M I$ body mass index, $C V D$ cardiovascular disease, $D B P$ diastolic blood pressure, $e G F R$ estimated glomerular filtration rate, $H b A 1 c$ hemoglobin A1c, $L D L$-c low-density lipoprotein cholesterol, $M I$ myocardial infarction, $S B P$ systolic blood pressure Mean and SD for 3B population-calculated on the basis of 3B data, mean and SD for other trials-reports from previous trials/publications

\section{DISCUSSION}

This study assessed the generalizability of four GLP-1 RA CVOTs (Dulaglutide-REWIND, Exenatide-EXSCEL, Liraglutide-LEADER, Semaglutide-SUSTAIN-6) to Chinese patients with T2DM. On the basis of six key I/E criteria, our findings suggest that REWIND would have enrolled the largest proportion of general Chinese patients with T2DM among the four CVOTs. REWIND performed better than other CVOTs in matching with the largest proportion of the 3B population on key baseline characteristics.

Several prior studies assessing the generalizability of the GLP-1 RA CVOTs to the general T2DM population worldwide have been conducted [21, 22, 32]. Boye et al. reported that REWIND was more generalizable to the US adult T2DM population than other GLP-1 RA CVOTs (42.6\%) [21]. Sciannameo et al. found that REWIND was most generalizable $(35.8 \%)$ to the general European T2DM population compared with other GLP-1 RA CVOTs [22]. Although the proportion of reference population eligible for REWIND is slightly lower in our study $(31.1 \%)$ compared to the US and Italian studies, our conclusion that REWIND is most generalizable to Chinese patients with T2DM among CVOTs is consistent with these studies, which corroborates the finding regardless of differences in geographical and cultural factors.

There have been reports of ethnic differences in response to GLP-1 RAs treatment between 
Table 3 Number and proportion of 3B population matched on the baseline characteristics of each CVOT population

\begin{tabular}{lllll}
\hline Baseline characteristics & REWIND [7] & EXSCEL [9] & LEADER [11] & SUSTAIN-6 [13] \\
\hline Age & $18,193(71.6 \%)$ & $22,680(89.2 \%)^{*}$ & $19,816(78.0 \%)$ & $20,244(79.7 \%)$ \\
Gender & $24,504(96.4 \%)^{*}$ & $20,935(82.4 \%)$ & $20,123(79.2 \%)$ & $21,424(84.3 \%)$ \\
BMI $\left(\mathrm{kg} / \mathrm{m}^{2}\right)$ & $22,340(87.9 \%)$ & $23,604(92.9 \%)$ & $23,735(93.4 \%)^{*}$ & $23,103(90.9 \%)$ \\
Duration of diabetes & $24,757(97.4 \%)$ & $25,073(98.7 \%)$ & $25,079(98.7 \%)$ & $25,125(98.9 \%)^{*}$ \\
HbAlc & $20,133(79.2 \%)$ & $19,870(78.2 \%)$ & $20,916(82.3 \%)^{*}$ & $20,916(82.3 \%)^{*}$ \\
eGFR $\left(\geq 60 \mathrm{~mL} / \mathrm{min} / 1.73 \mathrm{~m}^{2}\right)$ & $24,816(97.7 \%)$ & $23,647(93.1 \%)$ & $24,682(97.1 \%)$ & $25,411(100 \%)^{*}$ \\
SBP & $24,436(96.2 \%)$ & $24,167(95.1 \%)$ & $24,593(96.8 \%)^{*}$ & $24,520(96.5 \%)$ \\
DBP & $24,566(96.7 \%)$ & $25,039(98.5 \%)^{*}$ & $24,584(96.7 \%)$ & $24,457(96.2 \%)$ \\
LDL-c & $24,325(95.7 \%)^{*}$ & $23,546(92.7 \%)$ & $23,434(92.2 \%)$ & $24,250(95.4 \%)$ \\
CVD history & $21,782(85.7 \%)^{*}$ & $8457(33.3 \%)$ & $7532(29.6 \%)$ & $10,361(40.8 \%)$ \\
Prior MI & $6353(25.0 \%)^{*}$ & NA & $2772(10.9 \%)$ & $2587(10.2 \%)$ \\
$\geq 4$ characteristics & $22,568(88.8 \%)$ & $22,061(86.8 \%)$ & $22,950(90.3 \%)$ & $23,512(92.5 \%)^{*}$ \\
$\geq 8$ characteristics & $14,832(58.4 \%)^{*}$ & $14,080(55.4 \%)$ & $13,062(51.4 \%)$ & $14,308(56.3 \%)$ \\
$\geq 10$ characteristics & $9247(36.4 \%)^{*}$ & $3789(14.9 \%)$ & $3075(12.1 \%)$ & $4571(18.0 \%)$ \\
\hline
\end{tabular}

$B M I$ body mass index, $C V D$ cardiovascular disease, $D B P$ diastolic blood pressure, $e G F R$ estimated glomerular filtration rate, $H b A 1 c$ hemoglobin A1c, $L D L-c$ low-density lipoprotein cholesterol, $M I$ myocardial infarction, $S B P$ systolic blood pressure For means (age, BMI, duration of diabetes, SBP, DBP, LDL-c, and HbA1c), matching the enrollment characteristic was defined as mean \pm 1.96 times the standard deviation; For percentages (male gender, eGFR, CVD history, and prior MI), matching the enrollment characteristic was defined as the percentage $\pm 5 \%$

Asterisks indicate the largest proportion

Asians and Caucasians which may be ascribed to differences in the pathophysiology of T2DM, dietary habits, and genetic factors [33-35]. Notably, most of the patients recruited in the previous CVOTs were Caucasians; therefore, the Asian population was underrepresented. A meta-analysis suggests that the Asian subgroup may receive greater cardiovascular benefit from GLP-1 RAs; however, the results are limited by the small sample size [36]. Our study examined the generalizability of CVOTs to general Chinese patients with T2DM using three different approaches and found that the cardioprotective properties of GLP-1 RAs are most generalizable to the Chinese T2DM population in real-world clinical practice.

It is worth noting that the $3 \mathrm{~B}$ population has lower BMI, higher LDL, and lower proportions of pre-existing CVD or prior MI compared to the CVOTs. For example, the proportion of patients with CVD history is $22.6 \%$ in the 3B study, comparable to REWIND (31.4\%) and the global estimate $(32.2 \%)$, but much lower than the other CVOTs [37]. This study demonstrated that $89.4 \%$ of the 3B population matched the inclusion criteria of CVD history in REWIND, which is much higher than that in EXSCEL (33.3\%) [9], LEADER (29.6\%) [11], and SUSTAIN-6 (40.8\%) [13], suggesting that REWIND and the $3 \mathrm{~B}$ population were most closely matched on CVD history. This may have contributed to the generalizability of REWIND which accounted for I/E criteria more applicable to the general T2DM populations during study design compared to other CVOTs. Dulaglutide was approved by the US Food and Drug 
Administration (FDA) in early 2020 for the reduction of major adverse cardiovascular events in adult patients with T2DM and established CVD or multiple cardiovascular risk factors. This study has generated evidence further supporting that REWIND results are broadly generalizable to the Chinese T2DM population with established CVD or multiple cardiovascular risk factors, which could be used in clinical decision-making in the treatment of T2DM in China. Moreover, the 3B study illustrated that $72 \%$ of the T2DM population had hypertension, dyslipidemia, or both [24], suggesting that the majority of patients with T2DM in China are at high risk of developing cardiovascular events. The primary prevention of cardiovascular complications is an important factor to consider in antidiabetic treatment prescribing in Chinese patients with T2DM.

This study has several strengths. First, the study included four CVOTs of GLP-1 RAs currently available (or soon will be) in China. Second, the analysis employed three approaches in assessing the generalizability of CVOTs to the 3B population, enhancing the reliability of the results. Third, sensitivity analyses were performed and conclusions were consistent, which supported the robustness of the findings. Fourth, the 3B population were recruited from primary, secondary, and tertiary hospitals in all major geographic regions of China, which broadly represents the Chinese T2DM population [24].

Our study also has some limitations. First, although the $3 \mathrm{~B}$ population provides the most representative data available for patients with T2DM in China, the population size is still limited. Also, as the 3B study was conducted several years ago, the population may not represent the current population. Second, self-reported information (i.e., duration of diabetes) in the 3B study may suffer from recall bias.

In summary, this study assessed the generalizability of results of four GLP-1 RAs CVOTs to general Chinese patients with T2DM. Although none of the CVOTs could fully represent the Chinese T2DM population, the REWIND study of once-weekly dulaglutide is more generalizable than the other three CVOTs.

\section{ACKNOWLEDGEMENTS}

Funding. This study and journal's Rapid Service Fee is funded by Eli Lilly and Company.

Authorship. All named authors meet the International Committee of Medical Journal Editors (ICMJE) criteria for authorship for this article, take responsibility for the integrity of the work, and have given their approval for this version to be published.

Authors' Contributions. Linong Ji and Xiaoling Cai both contributed to the concept and design, statistical analysis, and writing of the manuscript.

Disclosures. Linong Ji received sponsorship for presentation and consulting fees from AstraZeneca, Merck, Novartis, Eli Lilly, Roche, Sanofi-Aventis and Takeda, and grants/research support from AstraZeneca, Bristol Myers Squibb, Merck, Novartis, and Sanofi-Aventis; no other affiliations or activities may have affected the submitted work. Xiaoling Cai has nothing to disclose.

Compliance with Ethics Guidelines. IRB approval was not required for this particular study as the analysis is based on data from previously conducted studies. The study was conducted in compliance with the principles in the Declaration of Helsinki.

Data Availability. The datasets generated during and/or analyzed during the current study are available from the corresponding author on reasonable request.

Open Access. This article is licensed under a Creative Commons Attribution-NonCommercial 4.0 International License, which permits any non-commercial use, sharing, adaptation, distribution and reproduction in any medium or format, as long as you give appropriate credit to the original author(s) and the source, provide a link to the Creative Commons licence, and indicate if changes were made. The images or other third party material in this article are 
included in the article's Creative Commons licence, unless indicated otherwise in a credit line to the material. If material is not included in the article's Creative Commons licence and your intended use is not permitted by statutory regulation or exceeds the permitted use, you will need to obtain permission directly from the copyright holder. To view a copy of this licence, visit http://creativecommons.org/licenses/by$\mathrm{nc} / 4.0 /$.

\section{REFERENCES}

1. Hossain ME, Uddin S, Khan A, Moni MA. A framework to understand the progression of cardiovascular disease for type 2 diabetes mellitus patients using a network approach. Int J Environ Res Public Health. 2020;17(2):596.

2. Smith RJ, Goldfine AB, Hiatt WR. Evaluating the cardiovascular safety of new medications for type 2 diabetes: time to reassess? Diabetes Care. 2016;39(5):738-42.

3. Hiatt WR, Kaul S, Smith RJ. The cardiovascular safety of diabetes drugs-insights from the rosiglitazone experience. N Engl J Med. 2013;369(14): 1285-7.

4. Home PD, Pocock SJ, Beck-Nielsen $\mathrm{H}$, et al. Rosiglitazone evaluated for cardiovascular outcomes in oral agent combination therapy for type 2 diabetes (RECORD): a multicentre, randomised, open-label trial. Lancet. 2009;373(9681):2125-35.

5. Dormandy JA, Charbonnel B, Eckland DJ, et al. Secondary prevention of macrovascular events in patients with type 2 diabetes in the PROactive Study (PROspective pioglitAzone Clinical Trial In macroVascular Events): a randomised controlled trial. Lancet. 2005;366(9493):1279-89.

6. Mendes D, Alves C, Batel-Marques F. Number needed to harm in the post-marketing safety evaluation: results for rosiglitazone and pioglitazone. Pharmacoepidemiol Drug Saf. 2015;24(12): 1259-70.

7. Gerstein HC, Colhoun HM, Dagenais GR, et al. Dulaglutide and cardiovascular outcomes in type 2 diabetes (REWIND): a double-blind, randomised placebo-controlled trial. Lancet. 2019;394(10193): 121-30.

8. Gerstein HC, Colhoun HM, Dagenais GR, et al. Design and baseline characteristics of participants in the Researching cardiovascular Events with a Weekly INcretin in Diabetes (REWIND) trial on the cardiovascular effects of dulaglutide. Diabetes Obes Metab. 2018;20(1):42-9.

9. Holman RR, Bethel MA, Mentz RJ, et al. Effects of once-weekly exenatide on cardiovascular outcomes in type 2 diabetes. N Engl J Med. 2017;377(13): $1228-39$.

10. Mentz RJ, Bethel MA, Gustavson S, et al. Baseline characteristics of patients enrolled in the Exenatide Study of Cardiovascular Event Lowering (EXSCEL). Am Heart J. 2017;187:1-9.

11. Marso SP, Daniels GH, Brown-Frandsen K, et al. Liraglutide and cardiovascular outcomes in type 2 diabetes. N Engl J Med. 2016;375(4):311-22.

12. Marso SP, Poulter NR, Nissen SE, et al. Design of the liraglutide effect and action in diabetes: evaluation of cardiovascular outcome results (LEADER) trial. Am Heart J. 2013;166(5):823-830.e825.

13. Marso SP, Bain SC, Consoli A, et al. Semaglutide and cardiovascular outcomes in patients with type 2 diabetes. N Engl J Med. 2016;375(19): 1834-44.

14. Kristensen SL, Rørth R, Jhund PS, et al. Cardiovascular, mortality, and kidney outcomes with GLP-1 receptor agonists in patients with type 2 diabetes: a systematic review and meta-analysis of cardiovascular outcome trials. Lancet Diabetes Endocrinol. 2019;7(10):776-85.

15. Cardiovascular Disease and Risk Management. Standards of medical care in diabetes-2020. Diabetes Care. 2020;43(Suppl 1):S111-s134.

16. Pharmacologic Approaches to Glycemic Treatment. Standards of medical care in diabetes-2020. Diabetes Care. 2020;43(Suppl 1):S98-s110.

17. Hernandez AF, Green JB, Janmohamed S, et al. Albiglutide and cardiovascular outcomes in patients with type 2 diabetes and cardiovascular disease (Harmony Outcomes): a double-blind, randomised placebo-controlled trial. Lancet. 2018;392(10157): 1519-29.

18. Husain M, Birkenfeld AL, Donsmark M, et al. Oral semaglutide and cardiovascular outcomes in patients with type 2 diabetes. $\mathrm{N}$ Engl J Med. 2019;381(9):841-51.

19. Pfeffer MA, Claggett B, Diaz R, et al. Lixisenatide in patients with type 2 diabetes and acute coronary syndrome. N Engl J Med. 2015;373(23):2247-57.

20. Siderowf AD. Evidence from clinical trials: can we do better? NeuroRx. 2004;1(3):363-71. 
21. Boye KS, Riddle MC, Gerstein HC, et al. Generalizability of glucagon-like peptide-1 receptor agonist cardiovascular outcome trials to the overall type 2 diabetes population in the United States. Diabetes Obes Metab. 2019;21(6):1299-304.

22. Sciannameo V, Berchialla P, Orsi E, et al. Enrolment criteria for diabetes cardiovascular outcome trials do not inform on generalizability to clinical practice: the case of glucagon-like peptide-1 receptor agonists. Diabetes Obes Metab. 2020;22(5):817-27.

23. Kou S, Cao JY, Yeo S, Holmes-Walker DJ, Lau SL, Gunton JE. Ethnicity influences cardiovascular outcomes and complications in patients with type 2 diabetes. J Diabetes Complic. 2018;32(2): 144-9.

24. Ji L, Hu D, Pan C, et al. Primacy of the 3B approach to control risk factors for cardiovascular disease in type 2 diabetes patients. Am J Med. 2013;126(10): 925.e911-922.

25. Zhang YQ, Li Y, Dong YG, et al. A nationwide assessment of blood pressure control and the associated factors in Chinese type 2 diabetes mellitus patients. J Clin Hypertens (Greenwich). 2019;21(11):1654-63.

26. World Health Organization. Definition, diagnosis and classification of diabetes mellitus and its complications. Part 1: diagnosis and classification of diabetes mellitus. Geneva: Department of Noncommunicable Disease Surveillance, World Bank; 1999.

27. Chinese Diabetes Society. China guideline for type 2 diabetes (2007 edition). Zhonghua Yi Xue Za Zhi . 2008;18(88):1227-1246.

28. Xie Q, Hao CM, Ji L, et al. ACEI/ARB underused in patients with type 2 diabetes in Chinese population (CCMR-3B study). PLoS ONE. 2015;10(2):e0116970.

29. Zhou X, Ji L, Ran X, et al. Prevalence of obesity and its influence on achievement of cardiometabolic therapeutic goals in Chinese type 2 diabetes patients: an analysis of the nationwide, cross-sectional 3b study. PLoS ONE. 2016;11(1):e0144179.

30. Huang Y, Li J, Zhu X, et al. Relationship between healthy lifestyle behaviors and cardiovascular risk factors in Chinese patients with type 2 diabetes mellitus: a subanalysis of the CCMR-3B STUDY. Acta Diabetol. 2017;54(6):569-79.

31. U.S. National Library of Medicine. ClinicalTrials.gov. https://clinicaltrials.gov/. Accessed Jan 8.

32. Hinton W, Feher M, Munro N, Walker M, de Lusignan S. Real-world prevalence of the inclusion criteria for the LEADER trial: data from a national general practice network. Diabetes Obes Metab. 2019;21(7):1661-7.

33. Cho YM. Incretin physiology and pathophysiology from an Asian perspective. J Diabetes Investig. 2015;6(5):495-507.

34. Kim YG, Hahn S, Oh TJ, Park KS, Cho YM. Differences in the HbA1c-lowering efficacy of glucagonlike peptide-1 analogues between Asians and nonAsians: a systematic review and meta-analysis. Diabetes Obes Metab. 2014;16(10):900-9.

35. Velásquez-Mieyer PA, Cowan PA, Pérez-Faustinelli $S$, et al. Racial disparity in glucagon-like peptide 1 and inflammation markers among severely obese adolescents. Diabetes Care. 2008;31(4):770-5.

36. Kang YM, Cho YK, Lee J, et al. Asian subpopulations may exhibit greater cardiovascular benefit from long-acting glucagon-like peptide 1 receptor agonists: a meta-analysis of cardiovascular outcome trials. Diabetes Metab J. 2019;43(4):410-21.

37. Einarson TR, Acs A, Ludwig C, Panton UH. Prevalence of cardiovascular disease in type 2 diabetes: a systematic literature review of scientific evidence from across the world in 2007-2017. Cardiovasc Diabetol. 2018;17(1):83. 